PROCEEDINGS OF THE

AMERICAN MATHEMATICAL SOCIETY

Volume 51, Number 1, August 1975

\title{
A METHOD OF COMBINING FIXED POINTS
}

\author{
ROGER WAGGONER
}

ABSTRACT. It is now well known that in the category of finite polyhedra the fixed point property is not preserved by the operations of suspension, Cartesian product, adjunction along a segment, and join. Thus far none of the examples given have involved polyhedra of dimension 2 . It is shown in this paper that two fixed points $x$ and $y$ of a self-map of a polyhedron $K$ can be combined in a certain way if a certain criterion is satisfied by the $f$-image of a path from $x$ to $y$. Several corollaries follow, one of which is that if $K$ is a finite simply connected 2-polyhedron with no local separating points, $H_{2}(K) \neq 0$, and $K$ has a 2 -simplex $\sigma$ such that $\pi_{1}(K-$ Int $\sigma, z)$ is cyclic, then $K$ fails to have the fixed point property. This eliminates many 2-dimensional polyhedra from consideration as examples.

1. Introduction. It is now well known that in the category of finite polyhedra the fixed point property is not preserved by the operations of suspension, Cartesian product, adjunction along a segment, and join. The reader may consult [1], [2], or [3] as general references. Thus far none of the examples given have involved spaces of dimension 2. It is shown in this paper that two fixed points $x$ and $y$ of a self-map $f$ of a polyhedron $K$ can be combined in a certain way if a certain criterion is satisfied by the $f$-image of some path from $x$ to $y$. This is an improvement of a result of Shih [4]. A corollary is that if $K$ is a finite simply connected 2-polyhedron with no local separating points, $\mathrm{H}_{2}(K) \neq 0$, and $K$ has a 2-simplex $\sigma$ such that $\pi_{1}(K-$ Int $\sigma, z)$ has a single generator, then $K$ fails to have the fixed point property. This eliminates many 2-dimensional polyhedra from consideration as examples.

A map $f: X \rightarrow Y$ is a continuous function from the space $X$ to the space $Y$. If $f: X \rightarrow X$ is a map, then $F(f)$ denotes the fixed point set of $f$, and $L(f)$ is the Lefschetz number of $f$. The Euclidean metric of $E^{m}$ will be denoted by $d$. If $x$ and $y$ are points of $E^{m}$, then $(x, y),[x, y]$, and

Received by the editors April 10, 1973 and, in revised form, May 12, 1974. AMS (MOS) subject classifications (1970). Primary 55C20, 55A20. index.

Key words and phrases. Fixed point property, Lef schetz number, fixed point 
$R[x, y]$ denote, respectively, the open interval from $x$ to $y$, the closed interval from $x$ to $y$, and the closed ray from $x$ through $y$. An $m$-ball in $E^{m}$ is a set $\left\{x \mid d\left(x, x_{0}\right) \leq r\right\}$ for some fixed $x_{0}, r$. An $n$-disk in $E^{m}$ is a homeomorph of an $n$-ball in $E^{n}$.

The following two preliminary lemmas are concerned with $n$-balls in $E^{n}$.

Lemma 1. Let $D$ and $D_{0}$ be n-balls in $E^{n}$ with $D_{0}$ centered at the origin. Suppose $f: D \rightarrow D_{0}$ is a map such that $f^{-1}(0)=\left\{x_{0}, y_{0}\right\}$ and $y_{0} \epsilon$ Int $D$. Then there is a bomotopy $G: D \times I \rightarrow D_{0}$ for which $G(x, t)=f(x)$ if $x \in \mathrm{Bd} D$ or $t=0$, and $G(x, t)=0$ iff $x=x_{0}$ or $x=y_{t}=t x_{0}+(1-t) y_{0}$.

Proof. Let $b: D \times I \rightarrow D \times I$ be a map satisfying:

(i) $b(x, t)=(x, t)$ if $t=0$ or $x \in \mathrm{Bd} D$.

(ii) $b\left(y_{0}, t\right)=\left(y_{t}, t\right)$.

(iii) $b\left(y_{t}, 1\right)=x_{0}$.

(iv) $b \mid(D \times I)-\left\{\left(y_{t}, 1\right)\right\} \rightarrow(D \times I)-\left(x_{0}, 1\right)$ is a homeomorphism. Let $r: D-\left\{y_{0}\right\} \rightarrow \mathrm{Bd} D$ be the radial projection, and let $p: D \times I \rightarrow D$ be the projection onto the first factor. Then define $G$ by:

$$
G(x, t)= \begin{cases}0 & \text { if }(x, t)=\left(y_{t}, t\right) \text { or if } x=x_{0}, \\ f p b^{-1}(x, t) & \text { if } t \leq 1 / 2, \\ f p b^{-1}(x, t) & \text { if } t \geq 1 / 2 \text { and }\left|f p b^{-1}(x, t)\right| \leq\left|f r p b^{-1}(x, t)\right|, \\ {\left[(2-2 t)+\frac{(2 t-1)\left|f r p b^{-1}(x, t)\right|}{\left|f p b^{-1}(x, t)\right|}\right] \cdot f p b^{-1}(x, t) \text { otherwise. }}\end{cases}
$$

Lemma 2. If $D \subset D_{1}$ are n-balls in $E^{n}$ with center $z$, and $g: D \rightarrow D_{1}$ is a map with two fixed points $y_{0} \in$ Int $D$ and $x_{0}$, then there is a homotopy $H: D \times I \rightarrow D_{1}$ such that $H(x, t)=g(x)$ for $x \in \mathrm{Bd} D$ or $t=0$, and $H(x, t)$ $=x$ iff $x=x_{0}$ or $x=y_{t}=t x_{0}+(1-t) y_{0}$.

Proof. Let $r$ be the radius of $D_{1}$. Define $f(x)=g(x)-x$. Then for some $D_{0}, f: D \rightarrow D_{0}$ as in Lemma 1. Define $G$ as in Lemma 1, and let

$$
\gamma(x, t)=\max \{|G(x, t)+x-z| / r, 1\} .
$$

Finally, define $H$ by

$$
H(x, t)=(G(x, t)+x-z) / \gamma(x, t)+z .
$$


2. For the remainder of the paper, $K$ will be a finite polyhedron with a given triangulation and $f: K \rightarrow K$ will be a map with finitely many fixed points, all contained in maximal simplices of $K$. There is no loss of generality in placing these conditions on $f$, since any $g: K \rightarrow K$ is homotopic to such an f. A homotopy will be constructed which "slides" a fixed point of $f$ along a broken line from one maximal simplex into another.

The carrier of a point $x$ of $K$ is $\sigma(x)$. Let $M=\{(x, y) \in K \times K \mid \operatorname{cl} \sigma(x)$ $\cap \operatorname{cl} \sigma(y) \neq \varnothing\}$. For $A \subset K$, a map $f: K \rightarrow K$ is a proximity map on $A$ if for all $x \in A,(x, f(x)) \in M$. It is shown in [4] that there is a map $\alpha: M \times I \rightarrow K$ satisfying:

(i) $\alpha(x, y, 0)=x$ and $\alpha(x, y, 1)=y$,

(ii) $\alpha(x, x, t)=x$ for all $t \in I$,

(iii) $\alpha(x, y, t) \neq x$ if $x \neq y$ and $t \neq 0$, and $\alpha(x, y, t) \neq y$ if $x \neq y$ and $t \neq 1$.

Lemma 3. Let $[a, b]$ be an interval in some maximal simplex $\sigma$ of $K$, $a \in$ Int $\sigma$ and $b \in \mathrm{Bd} \sigma$, such that $f$ is a proximity map on an $\epsilon$-neighborbood $U([a, b], \epsilon)=U$ of $[a, b]$ and $F(f) \cap U=\{a\}$. Then there is a homotopy $H$ : $K \times I \rightarrow K$ satisfying:

(i) $H(x, t)=f(x)$ if $t=0$ or $x \notin U$,

(ii) $H(x, t)=x$ for $x \in U$ iff $x=x_{t}=(1-t) a+t b, 0 \leq t \leq 1$.

Proof. For convenience suppose that the $\epsilon$-neighborhood of $a, U(a, \epsilon)$, is in Int $\sigma$. Let $\gamma: I \rightarrow I$ be a homeomorphism such that $\gamma(0)=0$ and for any $t \neq 1, a\left(f\left(x_{t}\right), x_{t}, \gamma(t)\right) \in$ Int $\sigma$. Let $R: U \rightarrow[a, b]$ be a retraction such that $R(x)=b$ iff $x \in U$ - Int $\sigma$. If $R(x)=x_{t}$, let $t_{x}=t$. For $x \in U$, write $x=r_{x} R(x)+\left(1-r_{x}\right) S(x)$ where $S(x) \in \operatorname{Bd} U$. Finally, define $H_{1}$ by

$$
I_{1}(x, s)= \begin{cases}f(x) & \text { if } x \notin U \\ \alpha\left(f(x), x, r_{x} \cdot \gamma\left(t_{x}\right)\right) & \text { if } x \in U \text { and } t_{x} \leq \sqrt{ } s \\ \alpha\left(f(x), x, r_{x} \cdot \gamma(\sqrt{ } s)\right) & \text { otherwise. }\end{cases}
$$

Choose a disk $D$ such that $[a, b] \subset D \subset \sigma \cap U$ and $[a, b] \cap \operatorname{Bd} D=\operatorname{Bd} \sigma \cap$ Bd $D=\{b\}$. For $x \in D$ let $g(x)=\alpha\left(f(x), x, \gamma\left(t_{x}\right)\right)$, and choose $D$ small enough so that $g(D) \subset \sigma$. Construct a map $H_{2}: D \times I \rightarrow \sigma$ as in Lemma 2 . Let $\beta: K \rightarrow I$ such that $\beta(x)=0$ if $x \notin U, \beta(x)=\gamma\left(t_{x}\right)$ if $x \in D$, and $0 \leq$ $\beta(x)<1$ otherwise. Extend $H_{2}$ to $K \times I$ by the definition $H_{2}(x, t)=$ $\alpha(f(x), x, \beta(x))$ if $x \in U-D$ and $H_{2}(x, t)=f(x)$ if $x \notin U$. Now define $H$ by 


$$
H(x, s)= \begin{cases}H_{2}(x, s) & \text { if } t_{x} \leq(s+\sqrt{ } s) / 2, \\ H_{1}(x, s) & \text { if } t_{x} \geq \sqrt{ } s \\ H_{2}\left(x, 2 s\left[\left(\sqrt{ } s-t_{x}\right) /(\sqrt{ } s-s)\right]\right) & \text { otherwise. }\end{cases}
$$

Note that if $x=x_{t}=s$ then $H(x, s)=x$ because of the way in which $H_{2}$ was defined. On the other hand, if $s \neq 0$ and $(s+\sqrt{ } s) / 2 \leq t_{x} \leq \sqrt{ } s$ then $H(x, s) \neq x$ because $2 s\left(\sqrt{ } s-t_{x}\right) /(\sqrt{ } s-s) \leq s$ and $t_{x}>s$.

Lemma 4. Let $b$ be a fixed point of the map $f: K \rightarrow K$. Then there is an interval $[k, c]$, a neighborbood $U$ of $[k, c]$, and a bomotopy $H: K \times I \rightarrow K$ satisfying:

(i) $H(x, t)=f(x)$ if $x \notin U$;

(ii) if $x \in U$ then $H(x, t)=x$ iff $x=x_{t}=(1-t) b+t c$.

Proof. Let $\epsilon>0$ such that if $d(x, y)<\epsilon$ then $(x, y) \epsilon M$. Choose $\delta>0$ satisfying the following conditions:

(i) If $d(b, y)<\delta$ then $d(y, f(y))<\epsilon$.

(ii) There is a map $\eta: K \times I \rightarrow K$ such that $\eta(x, t)=x$ if $d(x, b) \geq$ $2 t \delta, \eta(x, t)=b$ if $d(x, b) \leq t \delta$, and $\eta_{t}: K-V_{t} \rightarrow K-\{b\}$ is a homeomorphism, where $V_{t}$ is the $t \delta$-neighborhood of $b$.

(iii) No other fixed points of $f$ lie with in $2 \delta$ of $b$. Let $\beta_{t}: K \rightarrow[0,1]$ with $\beta_{t}^{-1}(0)=K-V_{2 t}$ and $\beta_{t}^{-1}(1)=V_{t}$. Define $f_{t}$ by

$$
f_{t}(x)= \begin{cases}\alpha\left(f(x), x, \beta_{t}(x)\right) & \text { if } d(x, f(x))<\delta \\ f(x) & \text { otherwise. }\end{cases}
$$

Define $b_{t}: K \rightarrow K$ by $b_{t}(x)=\eta_{t} f_{t} \eta_{t}^{-1}(x)$. Then $F\left(b_{t}\right)=F(f), b_{0}=f$, and $b_{t}\left(V_{t}\right)=b$. Now define $H$ by

$$
H(x, t)=\left\{\begin{array}{l}
b_{t}(x) \text { if } x \notin V_{t}, \\
(d(x, b) \cdot b) / t \delta+(1-d(x, b) / t \delta)[(1-t) t+t e] \text { otherwise }
\end{array}\right.
$$

where $[b, e] \subset K$. Choose a point $c$ on $[b, e]$ such that $d(b, c)=$ $(\delta \cdot d(e, b)) /(\delta+d(e, b))$. Then for $x \in V_{t}, H(x, t)=x$ iff $x \in[b, e]$ and $d(H(x, t), b)=d(x, b)$, which means $(1-d(x, b) / t \delta) \cdot(t d(e, b))=d(x, b)$; hence $d(x, b)=(t \delta d(e, b)) /(\delta+d(e, b))$. Thus $H(x, t)=x$ iff $x=(1-t) b+$ tc. 
Let $a$ and $c$ be fixed points for $f$. A regular combining homotopy from $a$ to $c$ is a map $H: K \times I \rightarrow K$ such that $H(x, 0)=f(x)$, and for $t \neq 0$, $H(x, t)=x$ iff $f(x)=x$ and $x \neq a$, or $x=p(\gamma(t))$ where $p: I \rightarrow K$ is a simple polygonal path from $a$ to $c$ and $\gamma: I \rightarrow I$ is an onto map with $\gamma\left(t_{1}\right) \leq \gamma\left(t_{2}\right)$ whenever $t_{1} \leq t_{2}$.

Recall that two fixed points $a$ and $c$ of $f$ are said to be equivalent if there is a path $p: I \rightarrow K$ from $a$ to $c$ such that $f p$ and $p$ are fixed endpoint homotopic. Two fixed points $a$ and $c$ of $f$ are said to be strongly equivalent if there is a simple polygonal path $p$ from $a$ to $c$ and a homotopy $G: I \times I \rightarrow K$ such that $G(s, 0)=f(p(s))$ and $G(s, t)=p(s)$ iff $t=1$ or $f p(s)=p(s)$. The relation of strong equivalence is an equivalence relation on the fixed point set of $f$.

Theorem 1. Let $K$ be a finite polybedron and let $f: K \rightarrow K$ be a map with finite fixed point set contained in the maximal simplices of $K$. The fixed points $a$ and $c$ of $f$ are strongly equivalent iff there is a regular combining bomotopy from a to $c$.

Proof. Suppose that $a$ and $c$ are strongly equivalent and let $p$ and $G$ be maps which satisfy the conditions of the definition. We may assume that the only fixed points of $f$ which lie on $p(I)$ are the points $a$ and $c$. Let $V$ be an open polygonal ne ighborhood (possibly infinite) of $p(I)-\{a, c\}$ with $\{a, c\} \subset \mathrm{Bd} V$. Choose $V$ in such a way that $\mathrm{Cl} V$ collapses to $p(I)$ with a map $r: \mathrm{Cl} V \rightarrow p(I)$, such that:

(i) if $x \in \mathrm{Cl} V$ then $\alpha(f(x), f r(x), t)$ is defined,

(ii) the ray $R[r(x), x]$ intersects $\mathrm{Bd} V$,

(iii) if $B(x)$ is the first point of the above ray on $\mathrm{Bd} V$, then the function $B$ is continuous.

Let $n(x)=d(x, B(x)) / d(r(x), B(x))$. Define $\hat{f}: K \rightarrow K$ by

$$
\hat{f}(x)= \begin{cases}f(x) & \text { if } x \notin V \\ \alpha(f(x), f r(x), 2 n(x)) & \text { if } 0<n(x) \leq 1 / 2 \\ G(r(x),(2-\delta)(n(x)-1 / 2)) & \text { otherwise. }\end{cases}
$$

If $\delta$ is chosen to be sufficiently small, $\hat{f}$ is a proximity map on $p(I)$. If $V$ is then chosen to be sufficiently small, $\hat{f}$ has the same fixed point set as $f$. Now the endpoints of $p(l)$ lie in maximal simplices of $K$. We may assume that $p(l)$ intersects nonmaximal simplices of $K$ in only finitely many points. In that case, Lemmas 3 and 4 demonstrate a method of "moving" the fixed 
point $a$ along $p(I)$. By composing a suitable homotopy between $f$ and $\hat{f}$ with those obtained from repeated applications of the lemmas, a regular combining homotopy from $a$ to $c$ can be constructed.

The converse is not necessary for what follows, and the proof is omitted.

3. Applications. In Theorem 2 below, a condition on $K$ is given which insures that any two equivalent fixed points of a map $f: K \rightarrow K$ be strongly equivalent. In that case, $f$ may be altered with a homotopy to obtain a map $f^{\prime}$ which has exactly one fixed point for each essential fixed point class. The proof of the preliminary lemma, although quite technical, is elementary and is omitted.

Lemma. Assume that $K$ bas no local separating points and let $a$ and $b$ be two fixed points of a map $f: K \rightarrow K$. Let $p$ be a patb from a to $b$ such that $f p(t) \neq p(t)$ for $t \neq 0,1$. Let $\left[t_{1}, t_{2}\right] \subset[0,1]$. Then there is a map $H: I \times I \rightarrow K$ satisfying:

(i) $H(t, 0)=f p(t)$, for all $t$,

(ii) $H(t, s)=p(t)$ iff $t=0,1$,

(iii) $\alpha(H(t, 1), p(t), s)$ is defined for all $t \notin\left[t_{1}, t_{2}\right]$.

Throughout this section it will be assumed that $K$ has no local separating points.

Theorem 2. Let $\sigma$ be a maximal simplex of $K$, and let $C$ be the cyclic subgroup of $\pi_{1}(K-\sigma)$ generated by a simple loop in $\mathrm{Bd} \sigma$. If the sequence

$$
0 \rightarrow C \rightarrow \pi_{1}(K-\sigma) \stackrel{i_{\#}}{\longrightarrow} \pi_{1}(K) \rightarrow 0
$$

is exact, where $i_{\#}$ is induced by inclusion, then any two equivalent fixed points of a map $f: K \rightarrow K$ are strongly equivalent.

Proof. Let $a$ and $b$ be equivalent fixed points of $f: K \rightarrow K$. Let $p$ be a simple path from $a$ to $b$ with $f p \sim p$. Let $\tau$ be a maximal simplex of some subdivision of $K$ with $a, b \notin \tau$ and $\tau \subset$ Int $\sigma$. We may assume that $p(I)$ meets Int $\tau$ in a simple arc. Let $\left[t_{1}, t_{2}\right] \subset[0,1]$ such that $p\left[t_{1}, t_{2}\right]=p(I) \cap \tau$. Use the preceding lemma to alter $f p$ with a homotopy to obtain a proximity map on $p(I)-p\left[t_{1}, t_{2}\right]$. Let $q$ be a path made up of a segment from $f p\left(t_{2}\right)$ to $y_{1} \epsilon$ Bd $\tau$, a path $\gamma$ in Bd $\tau$ from $y_{1}$ to $y_{2}$, and a segment from $y_{2}$ to $f p\left(t_{1}\right)$. Then $f\left[t_{1}, t_{2}\right] * q$ is a loop in $K$ - Int $\tau$. Now $f(p) * p^{-1} \sim f\left[t_{1}, t_{2}\right] * q$. Since $f(p) * p^{-1}$ is inessential in $K, f\left[t_{1}, t_{2}\right] * q$ is equivalent in $K-$ Int $\tau$ to $\alpha^{n}$, where $\alpha$ is a loop in $\mathrm{Bd} \tau$ which generates $C$. We may use a 
homotopy between $f\left[t_{1}, t_{2}\right] * q$ and $\alpha^{n}$ in $K$ - Int $\tau$, keeping $\gamma$ fixed throughout, to obtain a proximity map on $p(I)$. Notice that throughout the se homotopies the image of $p(t)$ is equal to $p(t)$ iff $t=0,1$. It follows that $a$ and $b$ are strongly equivalent.

Corollary 1. If, with the bypotheses of Theorem $2, \operatorname{dim} K \geq 3$, then any two equivalent fixed points of $f$ are strongly equivalent.

Proof. If $\operatorname{dim} \sigma \geq 3$, then $C=0$.

Theorem 1 and Corollary 1 give a result of Shih [4].

Corollary 2. If $\operatorname{dim} K=2, \pi_{1}(K)=0$, and for some $\sigma, \pi_{1}(K-$ Int $\sigma) \cong C$, then any two equivalent fixed points of $f$ are strongly equivalent. If, in addition, $\mathrm{H}_{2}(K) \neq 0$, then $K$ admits a map of Lefschetz number zero [5]. In this case the fixed points of $f$ may be eliminated completely.

Consider the following example. Let a be a simple polygonal loop in the real projective plane $\mathrm{RP}^{2}$ which generates $\pi_{1}\left(\mathrm{RP}^{2}\right)$. Attach a 2-cell $\sigma$ to $\mathrm{RP}^{2}$ by mapping $\mathrm{Bd} \sigma$ onto $\alpha$ with a map of degree 3. Call the resulting adjunction space $K$. It follows from Corollary 2 that $K$ fails to have the fixed point property.

Corollary 3 also follows immediately from Corollary 2.

Corollary 3. If $\operatorname{dim}(K)=2, \pi_{1}(K)=0, H_{2}(K) \neq 0$, and $\sigma$ is a 2-simplex such that $K \cap \sigma$ is a 1-simplex, then $K \cup \sigma$ fails to bave the fixed point property.

\section{REFERENCES}

1. R. H. Bing, The elusive fixed point property, Amer. Math. Monthly 76 (1969), 119-132. MR 38 \#5201.

2. R. F. Brown, The Lefschetz fixed point theorem, Scott, Foresman, Glenview, Ill., 1971. MR 44 \#1023.

3. E. R. Fadell, Recent results in the fixed point theory of continuous maps, Bull. Amer. Math. Soc. 76 (1970), 10-29. MR 42 \#6816.

4. Ken-hua Shih, On least number of fixed points and Nielsen numbers, Acta Math. Sinica 16 (1966), 223-232 = Chinese Math.-Acta 8 (1966), 234-243. MR 35 \#1004.

5. Roger Waggoner, A fixed point theorem for (n-2)-connected n-polyhedra, Proc. Amer. Math. Soc. 33 (1972), 143-145. MR 45 \#2699.

DEPARTMENT OF MATHEMATICS, UNIVERSITY OF SOUTHWESTERN LOUISIANA, LAFAYETTE, LOUISIANA 70501 